\title{
Preliminary results of lifetime measurements in neutron-rich ${ }^{53} \mathrm{Ti}$
}

Alina Goldkuhle ${ }^{1 *}$, Christoph Fransen ${ }^{1}$, Alfred Dewald ${ }^{1}$, Navin Alahari ${ }^{2}$, Marcel Beckers ${ }^{1}$, Benedikt Birkenbach ${ }^{1}$, Andrey Blazhev $^{1}$, Thomas Braunroth ${ }^{1}$, Emmanuel Clément ${ }^{2}$, Gilles De France ${ }^{2}$, Jérémie Dudouet ${ }^{3,4}$, Jürgen Eberth ${ }^{1}$, Herbert Hess $^{1}$, Bertrand Jacquot ${ }^{2}$, Yung-Hee Kim ${ }^{5}$, Antoine Lemasson ${ }^{2}$, Silvia Monica Lenzi ${ }^{6,7}$, Hongjie Li ${ }^{2}$, Joa Ljungvall ${ }^{3}$, Julia Litzinger ${ }^{1}$, Caterine Michelagnoli ${ }^{2,6,7}$, Claus Müller-Gatermann ${ }^{1}$, Daniel Ricardo Napoli ${ }^{8}$, Bondili Sreenivasa Nara Singh $^{9}$, Rosa Maria Perez-Vidal ${ }^{10}$, Damian Ralet ${ }^{3,11,12}$, Peter Reiter ${ }^{1}$, Maurycy Rejmund ${ }^{2}$, Andreas Vogt ${ }^{1}$, Nigel Warr ${ }^{1}$, Karl Oskar Zell ${ }^{1}$, Magda Zielińska ${ }^{13}$, and the AGATA-Collaboration

${ }^{1}$ Institut für Kernphysik, Universität zu Köln, 50937 Köln, Germany

${ }^{2}$ GANIL, CEA/DRF-CNRS/IN2P3, BP 55027, 14076 Caen Cedex 5, France

${ }^{3}$ CSNSM, Université Paris-Sud, CNRS/IN2P3, Université Paris-Saclay, F-91405 Orsay, France

${ }^{4}$ Université de Lyon, Université de Lyon 1, CNRS/IN2P3, IPN-Lyon, F-69622 Villeurbanne, France

${ }^{5}$ Institut Laue-Langevin, BP 156, 38042 Grenoble Cedex 9, France

${ }^{6}$ INFN Sezione di Padova, I-35141 Padova, Italy

${ }^{7}$ Dipartimento di Fisica e Astronomia dell’Università di Padova, I-35131 Padova, Italy

${ }^{8}$ Laboratori Nazionali di Legnaro, INFN, I-35020 Legnaro, Italy

${ }^{9}$ Nuclear Physics Group, Schuster Laboratory, University of Manchester, Manchester, UK

${ }^{10}$ GSI, Helmholtzzentrum für Schwerionenforschung GmbH, 64291 Darmstadt, Germany

${ }^{11}$ Institut für Kernphysik, Technische Universität Darmstadt, 64289 Darmstadt, Germany

${ }^{12}$ Instituto de Físcia Corpuscular, CSIC-Universidad de Valencia, E-46071 Valencia, Spain

${ }^{13}$ Irfu, CEA, Université Paris-Saclay, F-91191 Gif-sur-Yvette, France

\begin{abstract}
To study the nuclear structure of neutron-rich titanium isotopes, a lifetime measurement was performed at the Grand Accélérateur National d'Ions Lourds (GANIL) facility in Caen, France. The nuclei were produced in a multinucleon-transfer reaction by using a $6.76 \mathrm{MeV} / \mathrm{u}{ }^{238} \mathrm{U}$ beam. The Advanced Gamma Tracking Array (AGATA) was employed for the $\gamma$-ray detection and target-like recoils were identified event-by-event by the large-acceptance variable mode spectrometer (VAMOS++). Preliminary level lifetimes of the $\left(5 / 2^{-}\right)$ to $13 / 2^{-}$states of the yrast band in the neutron-rich nucleus ${ }^{53} \mathrm{Ti}$ were measured for the first time employing the recoil distance Doppler-shift (RDDS) method and the compact plunger for deep inelastic reactions. The differential decay curve method (DDCM) was used to obtain the lifetimes from the RDDS data.
\end{abstract}

\section{Introduction}

The nuclear structure in the region of neutron-rich Ti isotopes is of enhanced interest due to the observed features hinting at subshell closures at $N=32,34$ [1]. For example, in neutron-rich ${ }^{50-56} \mathrm{Ti}$ isotopes the staggering of the excitation energies for the $2_{1}^{+}$state points to emergence of subshells. In case of ${ }^{53} \mathrm{Ti}$, the excited states were reported by prompt $\gamma$-ray spectroscopy following $\beta$ decay [2]. The knowledge was extended by studies performed at the Gammasphere spectrometer [3] to identify the ${ }^{53} \mathrm{Ti}$ yrast cascade for the first time and to determine the excitation energy of states with spins up to $I^{\pi}=21 / 2^{-}$. In addition, the experimental yrast structure was compared with shellmodel calculations where the experimental data are particularly well described by calculations using the GXPF1 and GXPF1A interactions [3]. For the level energies this is directly related to the fact that the $v 1 f_{5 / 2}$ and $v 1 p_{1 / 2}$ orbitals are well separated from the $v 1 p_{3 / 2}$ orbital, a fact that also gives rise to the subshell closure at $N=32$. In con-

*e-mail: agoldkuhle@ikp.uni-koeln.de trast, calculations with the FPD6 Hamiltonian do not predict gaps at $N=32,34$. In order to verify the previous findings on shell closures, lifetimes of excited states are of great importance. From these, transition probabilities can be determined which represent important quantities to test the shell structure. In this work preliminary data of lifetimes for the $\left(5 / 2^{-}\right)$to $13 / 2^{-}$states of the yrast band of ${ }^{53} \mathrm{Ti}$ are presented for the first time.

\section{Experimental Details}

Excited states in neutron-rich titanium isotopes were populated as products of a multinucleon-transfer reaction induced by a ${ }^{238} \mathrm{U}$ beam at an energy of $E\left({ }^{238} \mathrm{U}\right)=$ $1608.9 \mathrm{MeV}(=6.76 \mathrm{MeV} / \mathrm{u})$ at the Grand Accélérateur National of d'Ions Lourds (GANIL) in Caen, France. The experimental setup consisted of the compact plunger for lifetime measurements of excited states produced in deep inelastic reactions [4] in combination with the Advanced Gamma Tracking Array (AGATA) [5] $\gamma$-ray spectrometer consisting of 2936 -fold segmented capsules in 10 
cryostats. A $1.5 \mathrm{mg} / \mathrm{cm}^{2}$ enriched ${ }^{50} \mathrm{Ti}$ plunger target was used, on which a natural copper fronting with a thickness of $0.4 \mathrm{mg} / \mathrm{cm}^{2}$ for heat conduction was evaporated. A ${ }^{\text {nat }} \mathrm{Mg}$ degrader foil with a thickness of $3.2 \mathrm{mg} / \mathrm{cm}^{2}$ was placed downstream from the target. Both the target and degrader foils were placed at an angle of $45^{\circ}$ with respect to the incoming beam which is close to the grazing angle of the multinucleon transfer reaction. Since the expected lifetimes in the neutron-rich titanium isotopes are a few picoseconds, the recoil distance Doppler-shift (RDDS) method [6] was employed. The target-like reaction products were detected and identified in the largeacceptance variable mode spectrometer VAMOS ++ [7-9]. These products exit the target foil with an average velocity $v_{T}$ resulting $\beta_{T}=v_{T} / c=12.7(2) \%$. The $\mathrm{Mg}$ foil degrades the energy of the ions to $3.68 \mathrm{MeV} / \mathrm{u}$ resulting in an average velocity of $\beta_{D}=11.7(2) \%$ that was measured directly by VAMOS++. $\beta_{T}$ was deduced from the experimental Doppler-shift between the two components of the transitions:

$$
E_{\mathrm{sh}}(\Delta \beta, \theta)=E_{\mathrm{us}} \cdot \frac{\sqrt{1-\Delta \beta^{2}}}{1-\Delta \beta \cdot \cos (\theta)},
$$

where $E_{\mathrm{sh}}$ is the energy of the fast component, i.e. the emission before the degrader, and $E_{\text {us }}$ is the intrinsic $\gamma$ ray energy of the degraded component, i.e. the emission after the degrader, which depends on the velocity difference $\Delta \beta=\beta_{T}-\beta_{D}$ as well as the polar angle $\theta$. During the experiment beam induced structural changes of the target prevented a determination of the distances between target and degrader, which are essential for a lifetime analysis. This problem could be solved by determining effective distances using precisely known lifetimes of ${ }^{46} \mathrm{Ti}$ [10]. Detailed information about the distance determination will be given in Ref. [11]. A total of six target-to-degrader distances, ranging from $130 \mu \mathrm{m}$ and $1000 \mu \mathrm{m}$, were set during the experiment, each with a measurement time of about 24 hours.

\section{Data analysis and results}

The reaction products were completely identified on an event-by-event basis using VAMOS++. The matrix of the energy loss obtained in the sum of the first three rows of the ionization chamber versus the total energy measured in the sum of all rows was used for the $Z$ identification. The mass $A$ and mass over charge $(A / Q)$ were obtained from the time-of-flight, the total energy and the magnetic rigidity $(B \rho)$. Further details will be given in Ref. [11]. After the particle identification Doppler-corrected $\gamma$-ray singles spectra could be generated for each nucleus by means of corresponding $Z$ and $A$ gates. The differential decay curve method (DDCM) [12] was employed for the lifetime analysis using the $\gamma$-ray singles spectra. Figure 1 shows the $\gamma$-ray statistics for ${ }^{53} \mathrm{Ti}$ in a sum spectrum in which the spectra of all six distances were added up. It was possible to identify nine transitions of ${ }^{53} \mathrm{Ti}$ (cf. Fig. 1) that are clearly visible after Doppler correction for the degraded component. These correspond to the decays of the first six

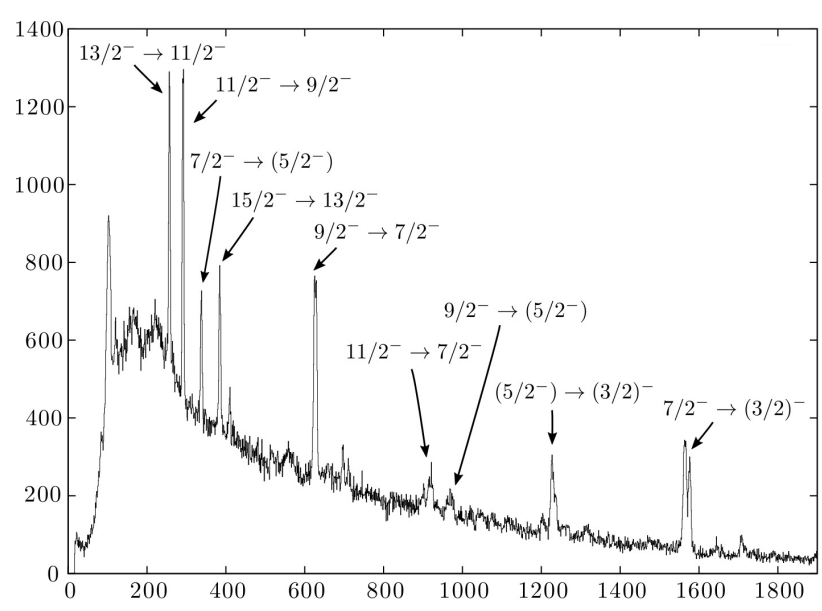

Figure 1. Doppler-corrected $\gamma$-ray spectrum in coincidence with ions identified as ${ }^{53} \mathrm{Ti}$, summed over all six distances. Nine $\gamma$-ray decays are observed.

excited states up to the $15 / 2^{-}$state of the yrast band in ${ }^{53} \mathrm{Ti}$. The level scheme in Fig. 2 contains only the transitions and levels observed in this work.

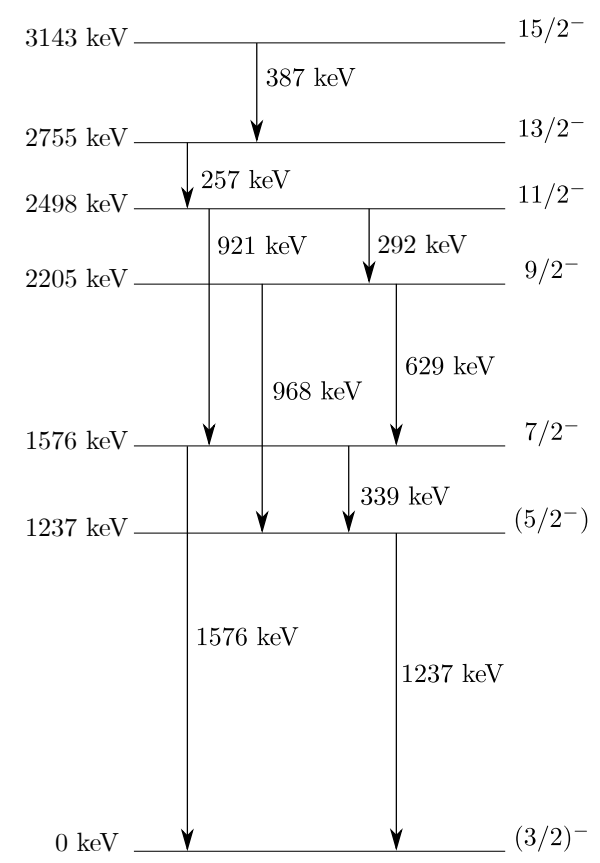

Figure 2. Partial level scheme of ${ }^{53} \mathrm{Ti}$ relevant for the present experiment.

For the evaluation of the $7 / 2^{-}$state the decay into the $(3 / 2)^{-}$state was used $(1576 \mathrm{keV})$, on the one hand due to the clear separation of the fast and degraded components, respectively, and on the other hand because it is by far the strongest decay branch. Furthermore, the $\gamma$-ray energy of the transition $7 / 2^{-} \rightarrow\left(5 / 2^{-}\right)$is significantly lower with $339 \mathrm{keV}$ and the Doppler shift is therefore smaller, which leads to a worse separation of the two components. In addition, the latter decay has a relative intensity of only 33 [13] normalized to the strongest decay with a relative intensity of 100 . Only the decay into the $7 / 2^{-}$state 

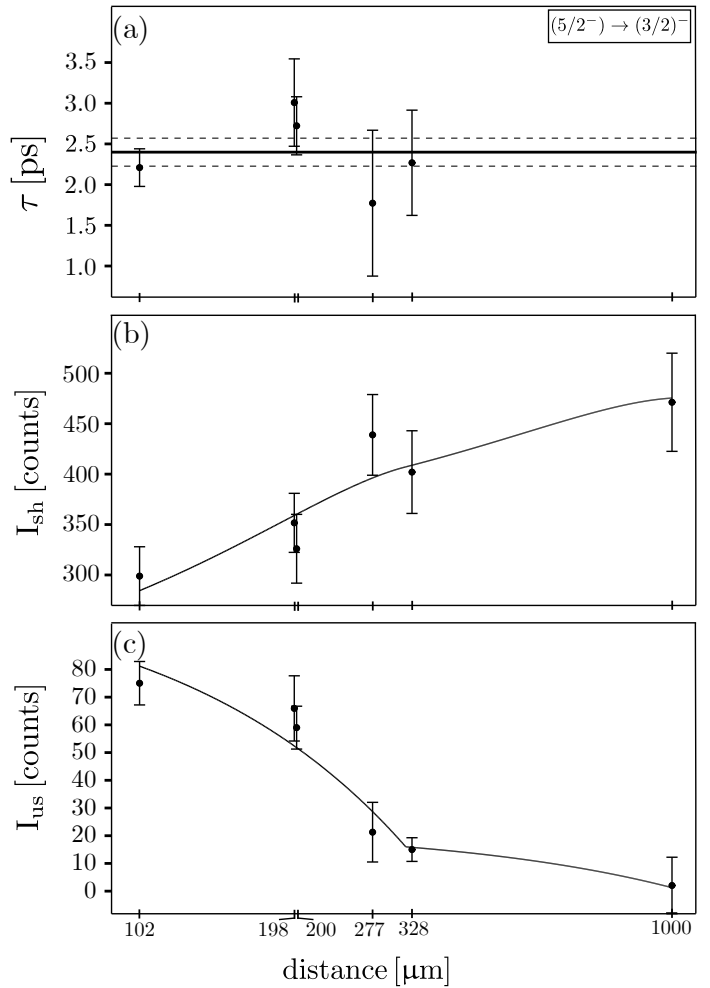
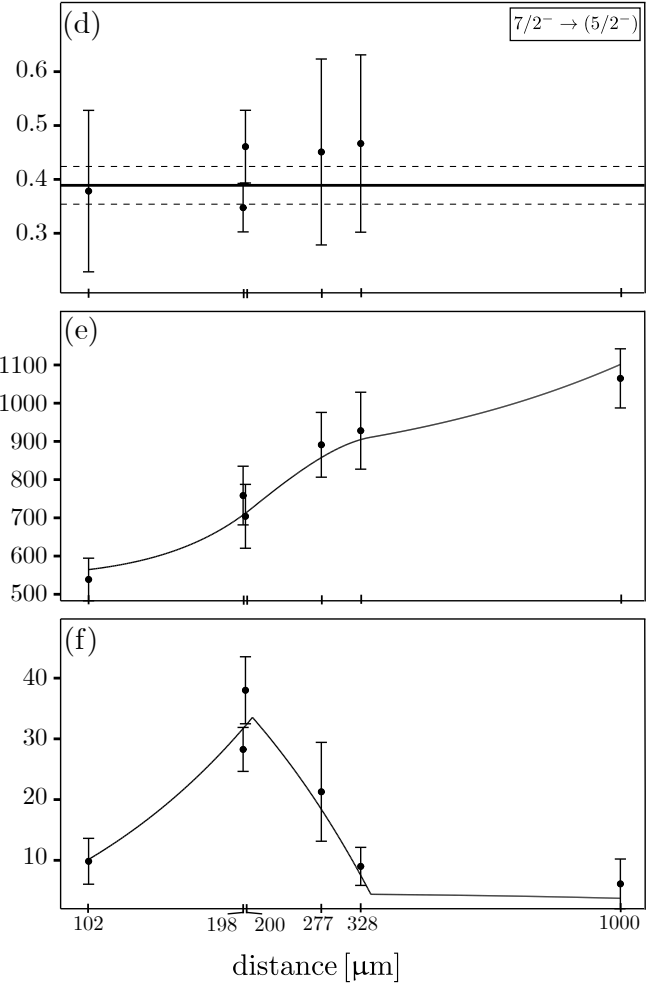

Figure 3. Plot of lifetime analysis for the $\left(5 / 2^{-}\right)$and $7 / 2^{-}$states in ${ }^{53} \mathrm{Ti}$. In the upper panels (a), (d) the black solid lines represent the weighted mean value of the lifetimes; dashed lines mark the statistical uncertainty. The intensities of the fast and degraded components are shown in (b),(e) and (c),(f), respectively. For the intensity determination of the degraded component (c),(f) a feeding correction was considered. Note the logarithmic distance scale.

$(629 \mathrm{keV})$ could be used to determine the lifetime of the $9 / 2^{-}$state, since this decay was much stronger with a relative intensity of 100 than the decay into the $\left(5 / 2^{-}\right)$ state $(968 \mathrm{keV}, 10.5)$. However, a smaller Doppler-shift is present due to the smaller transition energy. Although two transitions depopulate the $11 / 2^{-}$state, the transition to the $9 / 2^{-}$state with only $292 \mathrm{keV}$ is not suitable for the analysis, so the transition to the $7 / 2^{-}$state $(921 \mathrm{keV})$ was used even tough it only has a relative intensity of 17 as compared to the strongest decay branch with a relative intensity of 100 . Due to the insufficient separation of the $15 / 2^{-} \rightarrow 13 / 2^{-}$transition, it was not possible to evaluate the lifetime of the $15 / 2^{-}$state.

The peak positions and widths of the degraded components were fixed with calibrations of the strongest decay of ${ }^{50,52,53} \mathrm{Ti}$. The lifetime is extracted from intensities of the depopulating transition after subtraction of the observed feeding for each distance $x$ according to:

$$
\tau(x)=\frac{I_{\mathrm{us}}^{d}(x)-\alpha \cdot I_{\mathrm{us}}^{p}(x)}{\frac{d}{d x} I_{\mathrm{sh}}^{d}(x)} \frac{1}{v_{T}}
$$

S where $I_{\mathrm{us}}^{d}$ and $I_{\mathrm{sh}}^{d}$ are the efficiency-corrected and normalized intensities of the degraded and fast components of the depopulating $(d)$ and populating $(p)$ state, $\alpha$ includes the branching ratios and $v_{T}=\beta_{T} \cdot c$ is the mean velocity of the recoils before the degrader. According to DDCM, lifetime values are derived at each target-to-degrader distance and a constant line of lifetime values as a function of the distance is expected. Deviations from this constant line are indicators of systematic effects that can be detected using this method. Preliminary lifetime fits for the $\left(5 / 2^{-}\right) \rightarrow(3 / 2)^{-}$and $7 / 2^{-} \rightarrow(3 / 2)^{-}$transitions performed with the program NAPATAU [14] are shown in Fig. 3. Similar fits could also be generated for the higher lying states $9 / 2^{-}, 11 / 2^{-}$and $13 / 2^{-}$.

The middle panels (b),(e) show the intensities of the fast components of the depopulating transition and the lower panels (c),(f) those of the degraded components taking into account a feeding correction for observed feeders of the respective level of interest. As a result of these two, the deduced lifetime is shown in the upper panels (a),(d). The data are still under analysis, thus experimental preliminary results of measured lifetime values for the $\left(5 / 2^{-}\right)$to $13 / 2^{-}$states in ${ }^{53} \mathrm{Ti}$ are summarized in Table 1.

Table 1. Results of the preliminary lifetime values of the first five yrast states in ${ }^{53} \mathrm{Ti}$ from this work.

\begin{tabular}{cccc}
\hline \hline$I^{\pi}$ & $E_{\text {Level }}(\mathrm{keV})$ & $E_{\gamma}(\mathrm{keV})$ & $\tau(\mathrm{ps})$ \\
\hline$\left(5 / 2^{-}\right)$ & 1237.1 & 1237.1 & $2.4 \pm 0.5$ \\
$7 / 2^{+}$ & 1576.3 & 1576.3 & $0.4 \pm 0.4$ \\
$9 / 2^{-}$ & 2205.8 & 629.6 & $1.1 \pm 0.5$ \\
$11 / 2^{-}$ & 2498.1 & 921.8 & $3.5 \pm 0.7$ \\
$13 / 2^{-}$ & 2755.9 & 257.8 & $2.4 \pm 0.6$ \\
\hline \hline
\end{tabular}


Since the slowing down of the recoils in the degrader does not happen instantaneously, but takes a finite time, slowing down effects occur here (Doppler ShiftAttenuation (DSA)). These lead to the fact that the degraded peak has no longer a Gaussian shape, but a tail in the direction of the flight component, which should be assigned to the slow component, because the degrader is already reached. This can be problematic for the analysis of RDDS measurements with insufficient separation of the components, since these decays in the spectrum cannot be distinguished from those in which a recoil decays in flight between target and degrader. This effect depends on the lifetime and increases strongly with decreasing lifetime. In general, a correction for lifetimes smaller than $2 \mathrm{ps}$ is considered. This applies especially to the lifetimes of the $7 / 2^{-}$and $9 / 2^{-}$states for which such a correction must be made in a next step and is planned by means Monte-CarloSimulations using the G4LIFETIMEG toolkit [15].

\section{Summary}

Preliminary lifetime values of five excited states of the yrast band in ${ }^{53} \mathrm{Ti}$ were determined for the first time. The next step is to finalize the results by considering DSA effects for very short lifetimes. The results can be used to test shell-model predictions for different interactions. In addition to the existing calculations in Ref. [3], further interactions should be used to evaluate their predictability. With the new experimental transition strengths the description of the nuclear structure in ${ }^{53} \mathrm{Ti}$ may be improved. Further, the predicted evolution of shell closures at $N=32,34$ can be tested.

\section{Acknowledgements}

The authors would like to thank the staff at GANIL involved in this work for their support. Special thanks go to the operators for the excellent focusing of the beam. This work was supported by the German Research Foundation "Bundesministerium für Bildung und Forschung" (BMBF) under contract number 05P18PKFN9.

\section{References}

[1] D.C. Dinca, R.V.F. Janssens, A. Gade, D. Bazin, R. Broda, B.A. Brown, C.M. Campbell, M.P. Carpenter, P. Chowdhury, J.M. Cook et al., Phys. Rev. C 71, 041302 (2005)

[2] L.A. Parks, C.N. Davids, R.C. Pardo, Phys. Rev. C 15, 730 (1977)
[3] B. Fornal, S. Zhu, R.V.F. Janssens, M. Honma, R. Broda, B.A. Brown, M.P. Carpenter, S.J. Freeman, N. Hammond, F.G. Kondev et al., Phys. Rev. C 72, 044315 (2005)

[4] A. Dewald, O. Möller, P. Petkov, Progress in Particle and Nuclear Physics 67, 786 (2012)

[5] S. Akkoyun, A. Algora, B. Alikhani, F. Ameil, G. de Angelis, L. Arnold, A. Astier, A. Ataç, Y. Aubert, C. Aufranc et al., Nuclear Instruments and Methods in Physics Research Section A: Accelerators, Spectrometers, Detectors and Associated Equipment 668, 26 (2012)

[6] A. Dewald, O. Möller, P. Petkov, Progress in Particle and Nuclear Physics 67, 786 (2012)

[7] S. Pullanhiotan, M. Rejmund, A. Navin, W. Mittig, S. Bhattacharyya, Nuclear Instruments and Methods in Physics Research Section A: Accelerators, Spectrometers, Detectors and Associated Equipment 593, 343 (2008)

[8] M. Rejmund, B. Lecornu, A. Navin, C. Schmitt, S. Damoy, O. Delaune, J. Enguerrand, G. Fremont, P. Gangnant, L. Gaudefroy et al., Nuclear Instruments and Methods in Physics Research Section A: Accelerators, Spectrometers, Detectors and Associated Equipment 646, 184 (2011)

[9] M. Vandebrouck, A. Lemasson, M. Rejmund, G. Frémont, J. Pancin, A. Navin, C. Michelagnoli, J. Goupil, C. Spitaels, B. Jacquot, Nuclear Instruments and Methods in Physics Research Section A: Accelerators, Spectrometers, Detectors and Associated Equipment 812, 112 (2016)

[10] A. Goldkuhle, C. Fransen, A. Dewald, K. Arnswald, M. Bast, M. Beckers, A. Blazhev, T. Braunroth, G. Hackenberg, G. Häfner et al., The European Physical Journal A 55, 53 (2019)

[11] A. Goldkuhle et al. (2019), submitted for Phys. Rev. C

[12] A. Dewald, S. Harissopulos, P. von Brentano, Zeitschrift für Physik A Atomic Nuclei 334, 163 (1989)

[13] H. Junde, Nuclear Data Sheets 86, 2689 (2000)

[14] B. Saha, Computer code NAPATAU, Institute for Nuclear Physics, University of Cologne, unpublished

[15] S. Agostinelli, J. Allison, K. Amako, J. Apostolakis, H. Araujo, P. Arce, M. Asai, D. Axen, S. Banerjee, G. Barrand et al., Nuclear Instruments and Methods in Physics Research Section A: Accelerators, Spectrometers, Detectors and Associated Equipment 506, 250 (2003) 